Nota Científica

\title{
Relaciones biométricas y fecundidad de Glyphocrangon alata (Faxon, 1893) en la zona norte de Chile (21 $\left.{ }^{\circ} 19 ' S ; 7^{\circ} 20^{\prime} \mathrm{W}\right)$
}

\author{
Eduardo Quiroga J. y Raúl Soto M. \\ Departamento de Ciencias del Mar \\ Universidad Arturo Prat \\ Casilla 121, Iquique, Chile
}

\begin{abstract}
RESUMEN. Hembras ovígeras (41 especímenes) de Glyphocrangon alata, capturadas entre Iquique y Arica permitieron extender los rangos de distribución latitudinal y batimétrica de esta especie. Las relaciones entre longitud/ancho del caparazón y longitud del caparazón/longitud total son lineales en todas las tallas. La longitud del caparazón y la longitud total variaron entre 21,9-33,1 y 68,5-103,3 mm, respectivamente. La relación de fecundidad está descrita por una regresión lineal de la longitud/volumen del caparazón para un rango de 17 a 179 huevos. El volumen de loshuevos frescos fue de $15,68 \mathrm{~mm}^{3}$. Los huevos, pocos y grandes, caracterizan esta especie como de baja fecundidad y de desarrollo embrionario directo. El rendimiento reproductivo de 0,118 , se calculó sobre la base del peso húmedo; este valor es parecido al de los camarones carideos de regiones polares y templadas. Este estudio se efectuó dentro del Programa de desarrollo de las Naciones Unidas (PNUD).
\end{abstract}

Palabras claves: camarón de profundidad, Glyphocrangon alata, biometría, fecundidad

\section{Biometrics relationship and fecundity of Glyphocrangon alata (Faxon, 1893) in northern Chile (21 ${ }^{\circ} 19 ' S ; 7^{\circ} 20$ 'W)}

\begin{abstract}
Ovigerous females (41 specimens) from Glyphocrangon alata, captured between Iquique and Arica allowed to extend to the latitudinal and bathymetric distribution of the species. Relations carapace length/carapace width and carapace length/total length are linear at all morphological ranges. Carapace length and total varied between 21.933.1 and $68.5-103.3 \mathrm{~mm}$, respectively. A fecundity relation is described by a linear regression of carapace length/carapace volume for a range of 17 to $179 \mathrm{eggs}$; fresh-laid egg volume: $15.68 \mathrm{~mm}^{3}$. The eggs being big size and few characterize the species as having a low fecundity and a direct embryonic development. A reproductive output of 0.118 , based on wet weight values was calculated; it stands near the caridean shrimp values from polar and temperate regions. The study was derived from a report project from the United Nations Development Programme (UNDP).
\end{abstract}

Key words: deep-shrimps, Glyphocrangon alata, biometrics, fecundity.

\section{INTRODUCCION}

Se ha dentificado una gran variedad de especies de crustáceos decápodos provenientes de la zona preabisal y abisal en el área comprendida entre Arica e Iquique, los cuales han permitido ampliar el rango de distribución latitudinal y batimétrica de algunas especies y agregar nuevos registros para las aguas chilenas (Retamal y Soto, 1993). Entre las especies registradas, destaca Glyphocrangon alata (Faxon, 1893) camaron carídeo de profundidad, que se encuentra distribuido en el Pacífico este, desde Acapulco, Golfo de Panamá, Perú hasta Valparaíso, Chile (Méndez, 1981). Este camarón pertenece a la familia Glyphocrangonidae, la cual está representada en la zona norte de Chile por dos especies $G$. loricata y G. alata.

Existe escasa información acerca de la biología de estos camarones, especialmente con respecto a su biología reproductiva. El present trabajo tiene porobjetivo analizar en Glyphocrangon alata su fecundidad, el tamaño de los huevos, aspectos morfométricos, gravimétricos y comparar resultados con otros valores publicados para camarones de profunidad de la familia Crangonidae y Glyphocrangonidae. 


\section{MATERIALES Y METODOS}

Los especímenes fueron recolectados enel área Arica e Iquique, como parte del material obtenido durante el desarrollo del proyecto de aplicaciones de nuevas tecnologías para la captura de recursos hidrobiológicos marinos no tradicionales en la primera región (CHI/87/007), realizado por la Universidad Arturo Prat en 1990, con el patrocinio del Programa de Naciones Unidas para el Desarrollo (PNUD), destinado a evaluar la potencialidad de los recursos pesqueros bentónicos profundos del área, en busca de especies alternativas a la pesca tradicional.

Los ejemplares se obtuvieron en un lance de pesca efectuado con una red de arrastre de fondo a una profundidad entre los 470 y $700 \mathrm{~m}$, en los $70^{\circ} 20^{\prime} \mathrm{W}$ y $21^{\circ} 19^{\prime} \mathrm{S}$.

En este lance se recolectaron 41 hembras ovígeras, a las cuales se les extrajeron y contaron los huevos fijos al pleón. Con objeto de determinar los parámetros morfométricos y gravimétricos de esta especie se efectuaron las siguientes mediciones: longitud cefalotorácica (LC), longitud total (LT) y ancho cefalotorácico (AC), utilizando un vernier de $0,1 \mathrm{~mm}$ de precisión.

El peso total húmedo de la hembra sin huevos (WT) y el peso de los huevos, se obtuvo utilizando una balanza Sartorius de 0,05 gr de precisión y el tamaño de los huevos se midió utilizando una lupa con ocular graduado. Se estimó el rendimiento reproductivo (RO) en base al peso húmedo, aplicando la fórmula propuesta por Clarke et al. (1991): RO = (masa total de los huevos de la hembra/ masa de la hembra)*100.

\section{RESULTADOS}

\section{Biometría}

Las hembras recolectadas presentaron rangos de LC entre 21,9 y $33,1 \mathrm{~mm}$ y LT entre 68,5 y $103,3 \mathrm{~mm}$. Las relaciones morfométricas establecidas en G. alata quedaron definidas por las siguientes ecuaciones:

$\begin{array}{lcc}\text { LC-LT (Fig. 1): } & \text { LT }=4,5+3,03 \mathrm{~L} * \mathrm{C} & \mathrm{r}^{2}=0,92 \\ \text { LC-AC (Fig. 2): } & \text { AC }=1,33+0,73 * \text { LC } & \mathrm{r}^{2}=0,91 \\ \text { LT-WT (Fig. 3): } & \text { WT }=3,36 \mathrm{E}-5 * \mathrm{LT}^{2,8} & \mathrm{r}^{2}=0,72\end{array}$

\section{Tamaño de los huevos y fecundidad}

Los huevos se disponen fijos al pleón, formando racimos de color rojo-anaranjado, completamente vitelados (desarrollo embrionario incipiente). El tamaño promedio de los huevos, volumen promedio y volumen de la masa en huevos en G. alata es de 3, $12 \times 2,5 \mathrm{~mm}, 15,7 \mathrm{~mm}^{3}$ y $1286 \mathrm{~mm}^{3}$, respectivamente. Por otra parte, el número de huevos portados por la hembra varió entre 17 y 179 (Tabla 1). El número de huevos producidos se estimó a través de modelos de fecundidad (Figs. 4 y 5).

Tabla 1. Longitud cefalotorácica (LC) y número total de huevos en G. alata.

Table 1. Cephalothoraxic length (LC) and number of eggs of G. alata.

\begin{tabular}{|l|c|c|c|}
\hline Rangos & LC (mm) & $\begin{array}{r}\mathrm{N}^{*} \text { de } \\
\text { huevos }\end{array}$ & Autor \\
\hline MAX & 27,6 & 91 & Méndez, 1981 \\
MIN & 24,2 & 40 & Méndez, 1981 \\
MAX & 28,5 & 179 & Presente estudio \\
MIN & 24,4 & 17 & Presente estudio \\
\hline
\end{tabular}

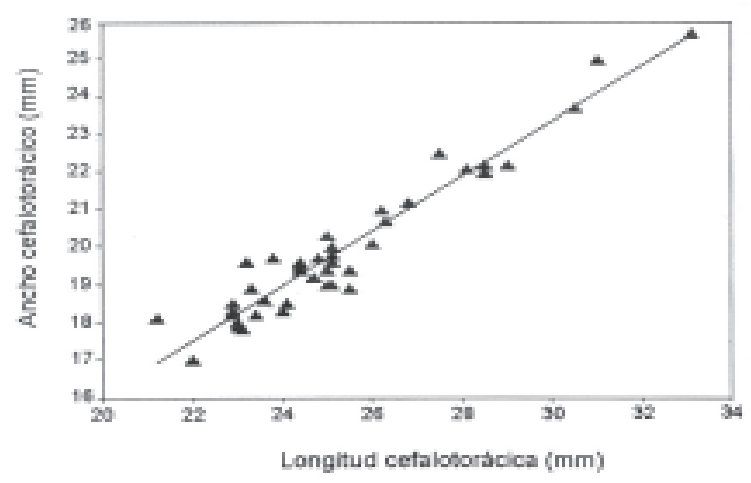

Figura 1. Relación entre la longitud cefalotorácica (LC) y el ancho cefalotorácico (AC) en G. alata: $\mathrm{AC}=1,33+0,73 * \mathrm{LC}\left(\mathrm{r}^{2}=0,92 ; n=41 ; \mathrm{P}<0,01\right)$. Figure 1. Cephalothoraxic length (LC)-cephalotoraxic width (AC) relationship for $G$. alata: $\mathrm{AC}=1,33+0,73 * \mathrm{LC}\left(\mathrm{r}^{2}=0,92 ; \mathrm{n}=41 ; \mathrm{P}<0,01\right)$. 


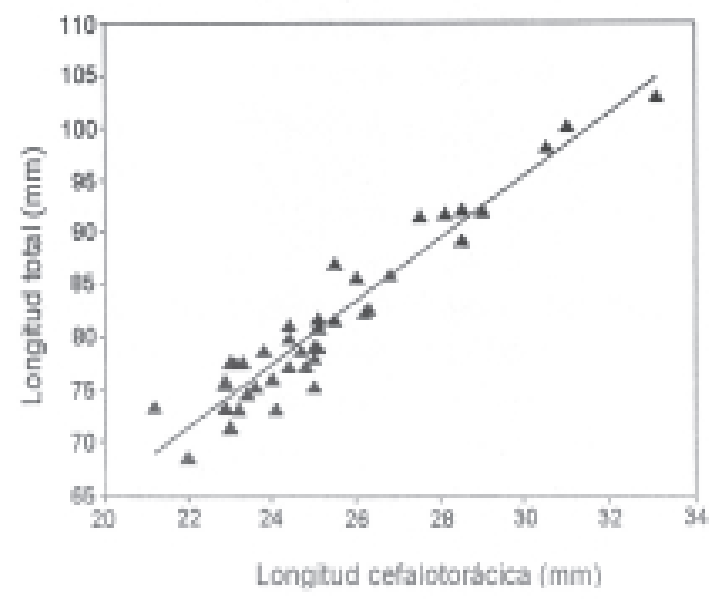

Figura 2. Relación entre la longitud cefalotorácica (LC) y la longitud total (LT) en G. alata: $\mathrm{LT}=4,5+3,03 * \mathrm{LC}\left(\mathrm{r}^{2}=0,91 ; \mathrm{n}=41 ; \mathrm{P}<0,01\right)$.

Figure 2. Cephalothoraxic length (LC)- total length (LT) relationship for $G$. alata:

$\mathrm{LT}=4,5+3,03 * \mathrm{LC}\left(\mathrm{r}^{2}=0,91 ; \mathrm{n}=41 ; \mathrm{P}<0,01\right)$.

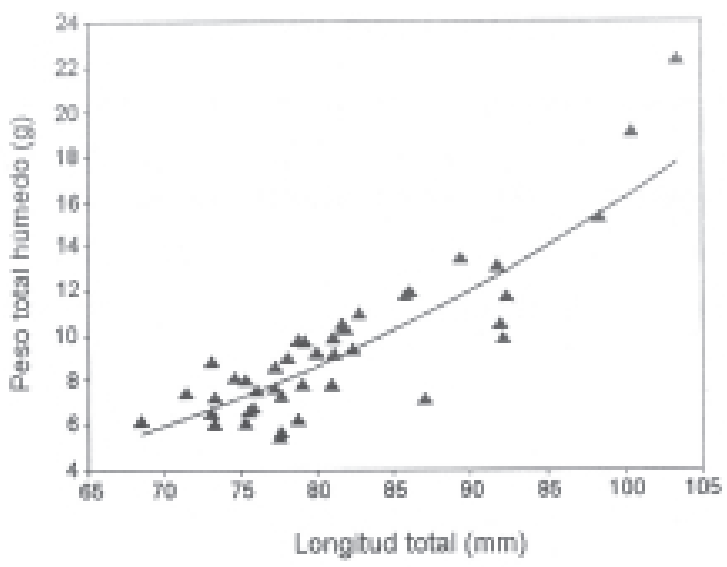

Figura 3. Relación entre la longitud total (LT) y el peso total húmedo (WT) en G. alata:

$\mathrm{WT}=3,36 \mathrm{E}-05^{*} \mathrm{LT}^{2.84}\left(\mathrm{r}^{2}=0,72 ; \mathrm{n}=41 ; \mathrm{P}<0,01\right)$.

Figure 3. Total length (LT)-wet weight (WT)

relationship for G. alata.

$\mathrm{WT}=3,36 \mathrm{E}-05 * \mathrm{LT}^{2.84}\left(\mathrm{r}^{2}=0,72 ; \mathrm{n}=41 ; \mathrm{P}<0,01\right)$.

\section{Rendimiento reproductivo}

En base al peso húmedo se estimó que el rendimiento rendimiento reproductivo (RO) de G. alata es de $0,118( \pm 0,014)$, el cual correspondió a un $11,8 \%$ del peso húmedo de la hembra.

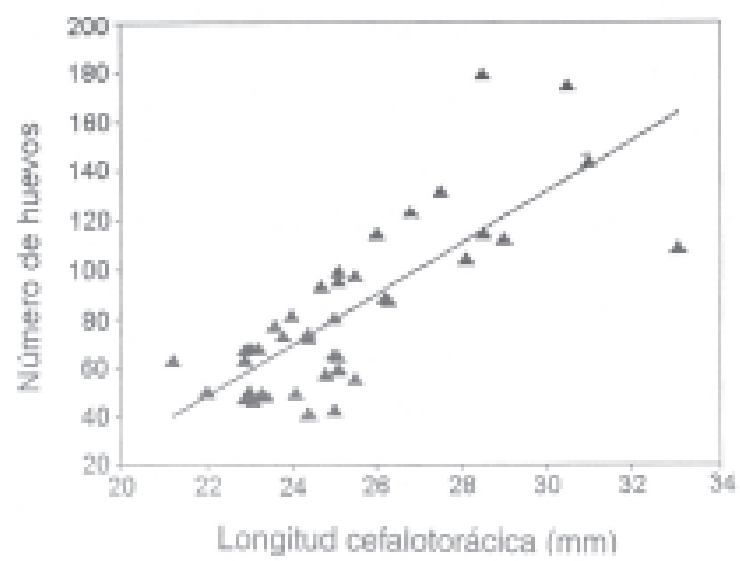

Figura 4. Relación entre la longitud cefalotorácica (LC) y el número de huevos (NH) en G. alata:

$\mathrm{NH}=-179,6+10,3 * \mathrm{LC}\left(\mathrm{r}^{2}=0,77 ; \mathrm{n}=41 ; \mathrm{P}<0,01\right)$.

Figure 4. Cephalothoraxic length (LC)-number of eggs (NH) relationship for $G$. alata:

$\mathrm{NH}=-179,6+10,3 * \mathrm{LC}\left(\mathrm{r}^{2}=0,77 ; n=41 ; \mathrm{P}<0,01\right)$.

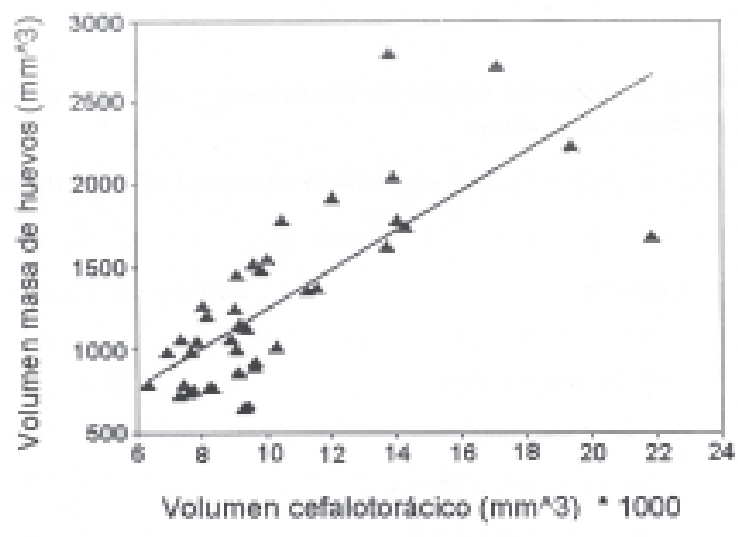

Figura 5. Relación entre el volumen cefalotorácico (VC) y el volumen masa de huevos (VMH) en G. alata: $\mathrm{VMH}=46,9+120,2 * \mathrm{VC}\left(\mathrm{r}^{2}=0,76 ; n=41 ; \mathrm{P}<0,01\right)$. Figure 5. Cephalothoraxic (VC)-volumen of egg-mass (VMH) relationship for G. alata.

$\mathrm{VMH}=46,9+120,2 * \mathrm{VC}\left(\mathrm{r}^{2}=0,76 ; \mathrm{n}=41 ; \mathrm{P}<0,01\right)$.

\section{DISCUSION}

Las hembras colectadas presentaron un rango mínimo y máximo de (LT) que osciló entre $68,5 \mathrm{~mm}$ y 103,3 mm, similar a lo registrado por Méndez (1981) de $36,6 \mathrm{~mm}$ y $107 \mathrm{~mm}$. Las relaciones biométricas LC-AC y LC-LT calculadas en $G$. alata presentan una clara relación positiva, indicando un crecimien- 
to isométrico, proporcional en todo el rango de tallas analizado.

El tamaño de los huevos encontrados en este estudio es característico de camarones de profundidad y muy similar a otras especies de zonas templadas y polares pertenecientes a la familia Crangonidae y Glyphocrangonidae (Tabla 2) . Debido a lo anteriormente expuesto y a lo planteado por Enríquez (1992), se puede establecer que en base a la eficiencia de las estrategias reproductivas, a mayores profundidades existiría una menor cantidad de alimento disponible para las larvas de crustáceos, las cuales son generalmente planctotróficas. Por esta razón, es más conveniente producir un menor número de huevos pero de mayor tamaño implementándolo con una mayor cantidad de vitelo con la tendencia a una incubación y un desarrollo abreviado y directo de los embriones. Esta relación ha sido descrita para camarones de los géneros Crangon y Sclerocrangon por Zarenkov (1965) y Makarov (1967).
En camarones carídeos, en general, se observa la existencia de una estrecha relación entre el tamaño de la hembra y el peso de los huevos (Ivanona y Vassilenko), 1987). Por su parte, Corey y Reid (1991) encuentran una alta relación entre el volumen del cefalotórax y el volumen de la masa de huevos y señalan que las especies de la familia Glyphocrangonidae se encuentran entre las de mayor volumen de cefalotórax versus volumen de la masa de huevos y número de huevos versus longitud del cefalotórax, describen de mejor forma la producción de huevos de esta especie.

El rendimiento reproductivo de G. alata basado en el peso húmedo $(0,118)$, presenta un valor cercano a lo descrito para otros camarones de aguas templadas y polares como Notocrangon antarticus $(0,118)$ y Pandalus montagui $(0,242)$ (Berenboy, 1982; Gorny et al., 1992).

Tabla 2. Tamaño medio de los huevos y número de huevos en diferentes especies de la familia Crangonidae y Glyphocrangonidae.

Table 2. Egg size and egg number means of the families Crangonidae and Glyphocrangonidae species.

\begin{tabular}{|l|c|c|c|}
\hline Especie & Tamaño del huevo $(\mathbf{m m})$ & No de huevos & Autor \\
\hline Sclerocrangon arrox & $3,33 \times 2,62$ & - & Zarenkov, 1965 \\
Sclenocrangon ferox & $3,00 \times 2,00$ & 133 & Zarenkov, 1965 \\
Scleracrangon zenkevirch & $3,72 \times 2,00$ & 52 & Zarenkov, 1965 \\
Notocrangon antarticus & $2,00 \times 1,52$ & 890 & Zarenkov, 1965 \\
Metacrangon variabilis & $2,30 \times 2,20$ & 29 & Zarenkov, 1965 \\
Glyphocrangon alata & $3,12 \times 2,50$ & 82 & Presente estudio \\
\hline
\end{tabular}

Tabla 3. Volumen medio del cefalotórax (VC) y volumen de la masa total de huevos (VMH) en diferentes especies de la familia Glyphocrangonidae.

Table 3. Cephalothoraxic mean volume (VC) and total egg-mass volume (VMH) of family Glyphocrangonidae species.

\begin{tabular}{|l|r|r|l|}
\hline Especie & VC $\left(\mathrm{mm}^{3}\right)$ & VMH $\left(\mathrm{mm}^{3}\right)$ & Autor \\
\hline Glyphocrangon longleyi & 6658,06 & 1224,26 & Reid y Corey, 1991 \\
Glyphocrangon sp. & 10535,66 & 743,40 & Reid y Corey, 1991 \\
Glyphocrangon alata & 10120,00 & 1285,76 & Presente estudio \\
\hline
\end{tabular}




\section{REFERENCIAS}

Berenboy, B. 1982. Reproduction of of the shrimp Pandalus borealis in the Barents Sea. Polar Mar. Fish. Oceanogr. Murmansk, 22 (1): 1-5.

Clarke, A., C. Hopkins y E. Nilssen. 1991. Eggs size and reproductive output in the deep-water prawn Pandalus borealis Kroyer, 1838. Functional Ecol. , 5: 724-730.

Corey, S. y D. Reid. 1991. Comparative fecundity of decapod Crustaceans. I. The fecundity of thirtythree species of nine families of caridean shrimp. Crustaceana, 60(3):271-294.

Enríquez, S. 1992. Estimación de fecundidad de algunos crustáceos decápodos marinos. semiario práctico. Curso magister en Ciencias y Oceanografía, Universidad de Concepción, 30 pp.

Gorny, M., W. Arntz, A. Clarke y D. Gore. 1992. Reproductive biology of caridea decapods from the Weddell Sea. Polar Biol. , 12: 111-120.
Ivanona, M. y S. Vassilenko. 1987. Relationship between number of eggs, brood weight, and female body weight in Crustacea. Int. Rev. ges. Hydrobiol., 72: 147-169.

Makarov, R. 1967. On the larval development of the genus Sclerocrangon G.O. Sars (Caridea, Crangonidae). Crustaceana, 2: 27-37.

Méndez, M. 1981. Clave de identificación y distribución de los langostinos y camarones (Crustacea: Decapoda) del mar y ríos de la costa del Perú. Bol. Inst. Mar, Perú-Callao, 5: 1-169.

Retamal, M.A. y R. Soto. 1993. Crustáceos decápodos abisales de la zona Iquique-Arica. Estud. Oceanol., 12: 1-8.

Zarenkov, N. 1965. Revision of the genera Crangon Fabricius and Sclerocrangon G. O. Sars (Decapoda, Crustacea). Zool. Zh. Moscow, 44: 1761-1775 (Traducido al ruso por Fish. Res. Bd. Canada, Transl. Service, 1465, 1970).

Recibido el 15 de julio d 1996.

Aceptado el 03 de enero de 1997. 